\title{
ESTUDIO EXPERIMENTAL DE PROCESOS DE CALENTAMIENTO Y ENFRIAMIENTO. RESULTADOS E IMPLICACIONES DIDÁCTICAS
}

\author{
FRAILE DELGADO, J.J. ${ }^{1}$, GARCÍA HOURCADE, J.L. ${ }^{2}$, GARCÍA MONTES, J.", \\ RODRÍGUEZ DE ÁVILA, C. ${ }^{2}$ y RUBIO REGUEIRO, J.L. ${ }^{2}$ \\ I IES Candeleda. Ávila. \\ 2 IES Mariano Quintanilla. Plaza Día Sanz, s/n. 40001 Segovia.
}

\begin{abstract}
SUMMARY
A detailed experimental study on different processes of heating and cooling allows to deduce that temperature variation, in these processes, in the course of time is not always the same, but it depends, to a great extent, on how the process is carried out. So to confuse the $\mathrm{T}-\mathrm{Q}$ relations (always lineal) with $\mathrm{T}$-t relations (dependent on the process) is a mistake that is necessary to avoid in our classes.
\end{abstract}

\section{INTRODUCCIÓN}

En los últimos años, en didáctica de las ciencias, se ha trabajado ampliamente para clarificar y explicitar los "errores conceptuales» o «ideas previas" con las que los alumnos se enfrentan a las situaciones de aprendizaje. Podemos decir que los trabajos de investigación que se han publicado en el ámbito español hacen referencia a prácticamente todos los aspectos que se abordan en los contenidos conceptuales de la física y química del actual segundo curso de BUP ( $y$, desde luego, los que son abordados en el $3^{\circ}$ y $4^{\circ}$ cursos de ESO).

Así, Ios relacionados con el tema de calor, temperatura y procesos térmicos han sido objeto de múltiples indagaciones y debates referidos fundamentalmente al «campo conceptual». (Michinel y D'Alessandro, 1994; Vázquez Díaz, 1987; Cervantes, 1987; Hierrezuelo et al., 1987; Fernández Uría, 1986; Sevilla Segura, 1986; Macedo de Burghi, 1985; García Hourcade y Rodríguez de Ávila, 1985).

Estos trabajos han servido tanto para clarificar las ideas que los alumnos poseen al empezar a estudiar estos temas (ya sıan explicaciones de fenómenos cotidianos o consecuencia de anteriores petíodos de instrucción), como para que los propios profesores profundicen su conocimiento acerca de lo que se entiende por calor.

La investigación en este campo ya ha tenido sus frutos, pues, si analizamos los libros de texto de física y química que se han publicado en los últimos años, nos daremos cuenta de que en ellos se hace especial hincapié en la diferenciación entre calor y temperatura, al definir el calor como la cantidad de energía transferida ( $y$ no contenida) en ciertos procesos o al retacionarlo con las variaciones de energía interna de los sistemas. Y es que, según nuestra opinión, la mejora en los procesos de enseñanza-aprendizaje tiene uno de sus pilares (no eI único) en la «ampliación y profundización» del conocimiento de los propios profesores.

Sin embargo, parece que se han investigado menos los propios precesos térmicos en sí; de hecho, no tenemos constancia de que se haya publicado ninguna indagación 
didáctica sobre cómo se enseñan, qué problemas suponen $u$ originan...

El trabajo que presentamos intenta evidenciar cómo la falta de reflexión, la rutina que preside muchas de nuestras clases y la excesiva atadura a los libros de texto se traduce en que, en muchos casos, homologuemos los procesos térmicos sin tener en cuenta cómo se producen, lo cual, desde nuestro punto de vista, constituye una fuente de error en el aprendizaje de nuestros alumnos, error que es fácilmente evitable si se ahonda en el estudio de tales procesos.

En nuestro caso, el disponer de un equipo LAO (laboratorio asistido por ordenador) nos ha permitido estudiar de forma exhaustiva los calentamientos y enfriamientos de diferentes sistemas y detectar problemas en los modos en que los venimos enseñando.

Las ventajas que reporta el LAO, tanto para la toma de datos como para su tratamiento gráfico, ha supuesto que podamos abordar análisis que sin disponer de este equipo hubieran revestido una excesiva dificultad o estarían llenos de imprecisiones.

Las conclusiones obtenidas resultan a veces sorprendentes y, desde luego, se alejan en muchos casos de lo que habitualmente solemos enseñar $u$ omitir en nuestras clases acerca de cómo se producen y desarrollan estos procesos.

\section{LOS PROCESOS TÉRMICOS Y LAS RELA- CIONES LINEALES}

El calor y los procesos térmicos constituyen uno de los contenidos importantes en la física y química del actual $2^{\circ}$ de BUP y también de $14^{\circ}$ curso de la nueva ESO. Todos los profesores sabemos que el grado de profundidad y la corrección con que se aborden los conceptos y procesos implicados será fundamental para poder enfrentarse a la termodinámica de niveles educativos superiores dentro de la enseñanza no universitaria.

Este tema suele comenzarse, frecuentemente, haciendo una reflexión sobre las diferencias entre $Q$ y $T$ y realizando actividades que favorezcan la asimilación correcta de tales nociones.

Al hablar de los «efectos del calor» trabajamos normalmente los cambios de temperatura y los cambios de estado (además de las dilataciones que no son objeto de estudio en este artículo). Es frecuente, por otra parte, que el estudio de las relaciones entre la variación de temperatura $(T)$ que experimenta un sistema y la energía que este mismo sistema ha recibido o perdido $(Q)$ se realice en el laboratorio de forma experimental, ya que además es una buena ocasión para trabajar uno de los procedimientos que estimamos más importantes dentro del área de las Ciencias Experimentales: el control de variables. En nuestro instituto y desde hace años, los alumnos de $2^{\circ}$ de BUP son enfrentados a la obtención experimental de la dependencia de la variación de temperatura que experimenta un cuerpo con la masa, el calor espećffico y la energía transferida.

Se trata, en suma, de poner a los alumnos en disposición de Ilegar a la expresión $Q=m . c_{e} . \Delta T$, expresión numérica que rige el comportamiento de todos los procesos de calentamiento y enfriamiento.

Lo que sucede es que, en realidad, no se realizan más que procesos de calentamiento (son más rápidos y más fáciles de llevar a cabo) y además, al no disponer de un procedimiento que permita medir directamente la energía comunicada al sistema que calentamos (p.e., una cierta masa de agua), lo que sugerimos a nuestros alumnos es que midan el tiempo que están calentando, ya sea con un mechero de gas o con una resistencia eléctrica (conviniéndoles, eso sí, para que no modifiquen la intensidad de la llama ni se intercambien las resistencias). En esta situación no controlamos $Q$ directamente (usamos una relación lineal entre Ia energía comunicada por una fuente y el tiempo que está funcionando) y medimos las variaciones de temperatura cada cierto intervalo de tiempo.

Por ello, es muy común que al analizar los resultados obtenidos se recurra a las representaciones gráficas (otro de los procedimientos básicos en Ciencias ExperimentaIes) y se represente $T\left({ }^{\circ} \mathrm{C}\right)$ frente a $t(s)$. Ahora bien, como el tiempo ha sido la variable que en el laboratorio nos ha permitido controlar la energía transferida $(Q)$, estas representaciones gráficas son asimiladas rápidamente a las que incluyen como variables $T\left({ }^{\circ} \mathrm{C}\right)$ y $Q$.

El resultados es que nuestros alumnos representan la variación de temperatura en un proceso de calentamiento de forma lineal, independiente de que en abscisas se utilice el tiempo o la energía. (Sin embargo, como veremos, la primera de las gráficas es siempre correcta mientras que la segunda no).

Es decir, se identifican gráficas como las que siguen:
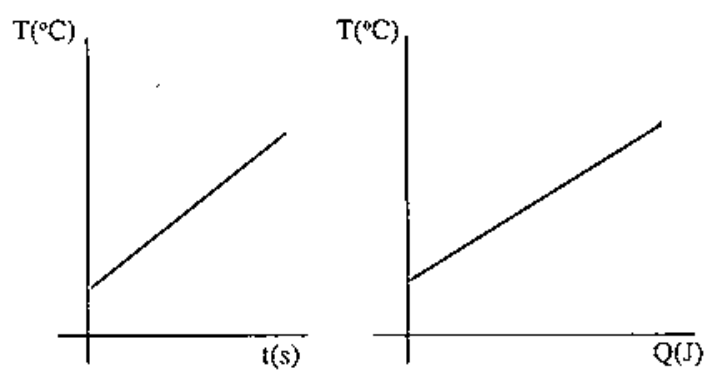

Estas relaciones lineales las solemos utilizar también nosotros, los profesores, al estudiar de forma cualitativa o cuantitativa procesos térmicos que incluyan cambios de estado, ya que son muy ilustrativas de estas situaciones. De nuevo, solemos usar indistintamente abscisas con valores de $t(s) \circ Q(J)$, ya que estamos mediatizados 
por las medidas que realizamos en el laboratorio (y que suponen que se comunica energía de forma constante con el tiempo). Así, no es difícil que en nuestras clases comentemos gráficas como la siguiente:
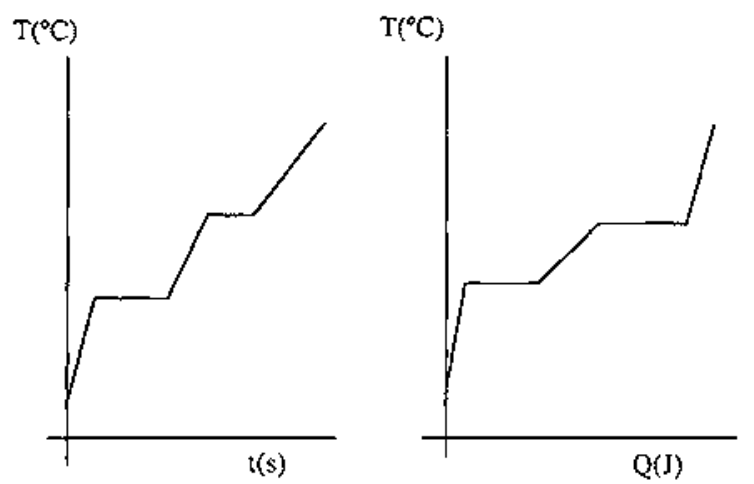

Este tipo de gráficas nos permite incluso llevar a cabo cálculos a partir de las mismas para la obtención de calores específicos o latentes.

Además, como la relación $\Delta T=Q /\left(m . c_{e}\right)=k \cdot Q$ se cumple independientemente del signo de $Q$ y el trabajo de laboratorio ha reforzado la idea de la relación lineal entre la temperatura y el tiempo, es bastante probable que los alumnos hayan asimilado que la relación $T / t(s)$ en un proceso de enfriamiento es también lineal, sin pararse a meditar (nosotros tampoco lo hacemos en muchas ocasiones) que el proceso de enfriamiento es totalmente distinto al de calentamiento con un mechero, que es con el que ellos han trabajado.

Esta homologación de los procesos de calentamiento y enfriamiento, así como la de todos los procesos de calentamiento entre sí, que implica dar por supuesto que en todos ellos se dan relaciones lineales $T\left({ }^{\circ} \mathrm{C}\right) / t(s)$ se pone de manifiesto cuando se estudian diversas situaciones. Así, por ejemplo, cuando utilizamos el método de las mezclas para determinar el calor específico (Ce) de un metal de forma experimental, al no profundizar en el estudio de cómo se lleva a cabo el calentamiento y el enfriamiento de las sustancias que se ponen en contacto, los alumnos piensan que la temperatura varía de forma lineal con el tiempo aun cuando el proceso que se sigue es totalmente diferente al que se da cuando se suministra energía de forma constante por medio de un foco térmi$\mathrm{co}$, que es lo que ellos han realizado de forma experimen. tal y para el que han comprobado que sí se cumple este tipo de dependencia.

Esta afirmación la fundamentamos, por una parte, con los resultados de un pequeño cuestionario que pasamos a los alumnos del instituto donde trabajamos y, por otra, presentando un análisis de algunos libros de texto que se han publicado en los últimos años y han sido escogidos sin otros criterios que el de ser de reciente publicación (despues del año 90, los de enseñanza no universitaria), ser de diferentes niveles educativos ( $2^{\circ}$ de BUP, ESO y transición a la universidad) y encontrarse en el departamento de física y química, enviados por unas u otras editoriales.

\section{Análisis del cuestionario}

Para indayzar sobre las ideas que nuestros alumnos tienen sobre cónı transcurren los procesos térmicos, elaboramos un pequeño cuestionario que presentamos en el anexo I.

La muestia sobre la que se llevó a cabo fue: todos los alumnos cle $2^{\circ}$ de BUP del centro (189) y los alumnos de $3^{\circ}$ de $\mathrm{BUJ}(98)$ y COU (78) que cursan una opción de ciencias en la que figure la física.

A la horia de analizar las respuestas se realizó una primera selección desechando como inválidos aquellos cuestionarios en los que se mezclaban de manera aleatoria diferentes tipos de relaciones entre la temperatura y el tiempo, o en los que se encontraban incorrecciones que denotaban un insuficiente manejo de las representaciones gráficas. De esta manera se rechazaron 47 cuestionarios correspondientes a $2^{\circ}$ de BUP, 22 a $3^{\circ}$ de BUP y 29 a CCU.

Se tomaron como válidos aquelios cuestionarios que incluían todas las relaciones lineales, o todas las no lineales. The esta manera se constató que 141 alumnos de $2^{\circ}$ de BUF $(74,6 \%), 73$ alumnos de $3^{\circ}$ de BUP $(74,5 \%)$ y 28 de COU $(35,8 \%)$ expresaron que las relaciones $T\left({ }^{\circ} \mathrm{C}\right) / t(s)$ en los diferentes procesos que se presentaban eran lineales, sin distinguir que algunos discurrían de manera totalmente distinta a otros.

Si los porcentajes se llevaran a cabo respecto de los alumnos cuyas respuestas son aceptadas (los alumnos cuyas resfiuestas no fueron consideradas válidas, desde luego, no habían tenido un proceso de aprendizaje correcto), éstos serían de un $99,3 \%$ en $2^{\circ}$ de BUP, de un $96 \%$ en $3^{\circ}$ de BUP y de un $57,1 \%$ en COU.

Solamente 1 alumno de $2^{\circ}, 3$ de $3^{\circ}$ y 21 de COU representaron las relaciones $T\left({ }^{\circ} \mathrm{C}\right) / t(s)$ como no lineales de forma cualitativamente correcta (era lo que se pedía), excluyendo de sus respuestas las relaciones lineales.

Estos resultados nos dan pie para pensar que mayoritariamente r!uestros alumnos han asimilado que, cuando un cuerpo se enfría o se calienta, la temperatura varía linealmenie con el tiempo de duración del proceso, independientemente de que sea enfriamiento o calentamiento y sin distinguir tampoco cómo se produzca éste.

Las razones por las cuales, conforme progresamos en el nivel educativo, aumentan los alumnos que expresan esas relaciones como no lineales nos son desconocidas (no era nuestro objetivo y quizás debiera ser objeto de una indagación). Puede, sin embargo, pensarse que tal hecho sea originado por el contacto, que en los niveles superiores ya ha comenzado a ser frecuente, con otros 
procesos no lineales (gráficas $P \cdot V$ en termodinámica, variación de la intensidad de campos gravitatorio y electrostático, disminución de la intensidad luminosa por absorción...) y que han podido transferirse sin realmente ser más que una intuición.

En cualquier caso, son los alumnos de $2^{\circ}$ y $3^{\circ}$ de BUP los que reflejan de forma más fiable los resultados del aprendizaje, ya que en $2^{\circ}$ el tema es objeto de estudio explícito y en $3^{\circ}$ aún no se ha cntrado en contacto con procesos no lineales, excepto los relativos a la variación de la posición con relación al ticmpo en los movimientos uniformemente acelerados. Estos atumnos muestran una mayoritaria e inequívoca concepción de los procesos térmicos en los que no caben más que relaciones lineales $T\left({ }^{\circ} \mathrm{C}\right) / \mathrm{t}(\mathrm{s})$.

\section{Análisis de los libros de texto}

Desde la perspectiva con que afrontamos nuestro estudio nos interesaba conocer si en los libros de texto que tratan este tema se explicitan las diversas formas en que se pueden dar los calentamientos y las diferencias con los enfriamientos, así como analizar, en los casos que aparezcan, las gráficas que se usan para ilustrar o representar esos procesos.

Ambos aspectos pueden servir para contrastar nuestra hipotesis acerca de cómo se enseñan los procesos térmicos.

Nosotros pensamos que es muy común en los libros de tex to (y en nuestras clases) obviar la meditación sobre los diferentes modos de calentarse o enfriarse un sistema, favoreciendo así un aprendizaje en el que todos los procesos térmicos suponen relaciones lineales de la temperatura $(T)$ con el tiempo $(t)$ de calentamiento o enfriamiento. Se confunden, en esta situación, relaciones matemáticas lineales $\Delta T\left({ }^{\circ} \mathrm{C}\right) / Q(J)\left[\Delta T=Q / m . c_{i}\right]$ con otras relaciones que no lo son, como la $T\left({ }^{\circ} \mathrm{C}\right) / t(s)$, que depende del proceso que se siga.

En algunas ocasiones este defecto en la presentación de los procesos térmicos pasa por una falta de tratamiento gráfico (ed. Anaya, 1995; ed. Bruño,1995), 10 que puede ser interpretado como desinterés por la manera en que evolucionan los sistemas en un proceso de calentamiento o enfriamiento.

En otros casos, y aun cuando se utilizan gráficas, se cometen algunos ertores. Es el caso del texto Proyecto Esla (texto encomiable, ya que trabaja de forma explícita las representaciones gráficas) de 1990 , que, cuando utiliza las gráficas para representar los procesos de calentamiento y enfriamiento, no establece diferencia alguna entre utilizar en el eje de abscisas $Q \circ t$. Un caso que no of rece ninguna duda sobre este confusionismo es la gráfica que este texto presenta para ilustrar el equilibrio térmico que alcanzan dos cuerpos a diferentes temperaturas puestos en contacto, y en la que ambos procesos son representados por rectas que convergen en el tiempo hasta la temperatura de equilibrio.
Sifuaciones semejantes de identificación de gráficas de procesos térmicos con abscisas $Q$ o $t$ sin ningún tipo de comentario o precisión sobre los mismos aparecen también en los libros de las editoriales Ecir (1991), Edelvives $(1990,1995)$ y Santillana (1995) que han sido analizados.

Tampoco los libros de niveles superiores, utilizados por alumnos aventajados de COU o de primer año de universidad, evitan en su tratamiento la confusión planteada entre relaciones $T / Q$ y $T / t$. Así, Fidalgo y Fernánde $z$ (1990) y Holton y Roller (1972) plantean relaciones lineales $T / t$ sin meditar sobre el tipo de proceso (calentamiento o enfriamiento) y la forma en que se lleva a cabo.

Reproducimos, para terminar este apartado, un párrafo que aparece en el capítulo 18 del excelente libro de Holton y Roller (1972) y que sirve para corroborar nuestras afirmaciones:

«[...] coloquemos 0,5 kilos de $\mathrm{H}_{2} \mathrm{O}$ y un termómetro en una vasija e introduzcamos la vasija en una mezcla congelante hasta que el agua se congele y continúe enfriándose muy por debajo de su punto de congelación, por ejemplo, hasta $-20^{\circ} \mathrm{C}$. Quitemos cntonces el vaso y coloquémosle en una habitación templada y, a intervalos iguales de tiempo convenientes anotemos la temperatura del hielo cuando se va calentando hasta que alcance el punto de fusión y funda. Si se construyc una gráfica de temperatura en función del tiempo, se obtendrá una curva como la siguiente :

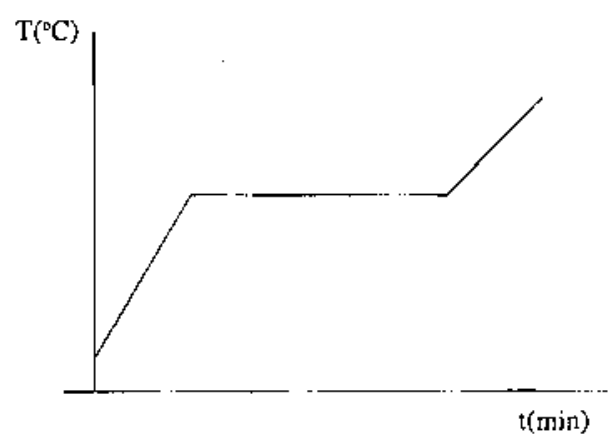

\section{EL ENFRIAMIENTO DE NEWTON}

Cuando un cuerpo se enfría en un medio a temperatura constante e inferior, el proceso térmico que se da es complejo y la pérdida de energía que origina el enfriamiento es una difícil superposición de fenómenos de radiación, convección y conducción.

En cualquier caso, tal proceso de enfriamiento se produce debido a una transferencia energética del cuerpo al ambiente originada por la diferencia de temperaturas entre ambos y cuya velocidad depende del gradiente de temperatura entre el cuerpo y el ambiente. Esto fue 
estudiado por Newton y se conoce en ja literatura como la ley del enfriamiento de Newton (Palacios, 1958; Català, 1961; Tipler, 1994).

No hemos encontrado más referencias explícitas a dicha Iey. El proceso de enfriamiento de un cuerpo se obvía en la mayoría de los textos consultados y se tratan, con generalidad y mayor o menor detalle, los de conducción, convección y radiación, haciendo referencia a la ley de Fourier en los casos de convección y conducción en regímenes estacionarios, es decir, cuando las temperaturas entre las que se transfiere energía, se mantienen constantes.

Obviamente, ése no es el caso de un cucrpo que se enfría (ni el de uno que se calienta en un ambiente a mayor temperatura) y quizás su aparente sencillez ha llevado al desinterés que encontramos en los libros de tex to universitarios por tales procesos.

La expresión matemática de la velocidad de enfriamiento sería:

$$
\Phi=-K \cdot A \cdot\left(T-T_{a}\right)
$$

donde $\Phi$ representa la variación energía (calor, $Q$ ) con respecto al tiempo $(d Q / d t) ; A$, la superficie del cuerpo que se enfría; $K$, un coeficiente de proporcionalidad; $T$, la tcmperatura del cuerpo que se enfria; y $T_{a}$, la temperatura ambiente.

Una prueba de lo que sospechamos (el desinterés por aparente sencillez o evidencia de esta ley), la da el hecho de que los tres textos que la explicitan denominan al coeficiente $K$ de distinta manera, denominaciones que van más aljá del nominalismo e implican, creemos, diferencias en la consideración del proceso. Así, Palacios (1948) la denomina «coeficiente de conductividad exterior»; Català (1961) se refiere a esta constante como "coeficiente de radiación» y Tipler (1994) lo llama «coeficiente superficial de transmisión del calor».

Todo ello nos hace pensar que el enfriamiento (y el calentamiento «natural») no ha sido muy detenidamente estudiado y que, en todo caso, como deciamos al principio de este epigrafe, es un fenómeno muy complejo que involucra las tres formas de trasmision del calor.

Creemos, y nuestros resultados así lo avalan, que la ley de Newton es una ley đe validez muy general y de más amplio margen de aplicación que to relatado generalmente por la literatura ${ }^{1}$.

Si en la ecuación 1 ponemos que $Q=m \cdot c_{c} \Delta T$, ley de validez general, y que expresa la relación lineal entre la energía perdida (enfriamiento) o ganada (calentamiento) y la variación de temperatura experimentada por el sistema, podremos poner:

$$
\frac{m c_{e} d T}{d t}=-K A\left(T-T_{a}\right)
$$

Dado que nuestras mediciones han sido siempre llevadas a cabo coll una cierta cantidad de agua aislada térmicamente, la temperatura exterior será prácticamente muy poco superior a la del ambiente, con lo que la «capa límite» đe convección puede estimarse despreciable. Al mismo tie mpo la radiación cumpliría las condiciones de desprecialsle diferencia térmica con el exterior, con lo que la energía radiada obedecería a un régimen «newtoniano".

En estas condiciones, la transferencia de energía es un proceso fundamentalmente de conducción a través de un aislante $y$, en este caso, la constante $K$ de (2) debe incorporal un factor $1 / d$, siendo $d$ la anchura del "calorífugo». E⿰氵工 análisis dimensional del término (K.A)/ $\left(d . m . c_{e}\right)$ que aparecería como una nueva constante nos dice que sus dimensiones son de $s^{t}$ si la constante $K$ es la de conducción térmica, lo que hace coherente dimensionalmente la ecuación siguiente:

$$
\frac{d T}{\left(T-T_{a}\right)}=-k d t \quad(3) \text { donde } k=(K A) /\left(d . m . c_{c}\right)
$$

Es ésta una sencilla ecuación diferencial cuya solución, si tomamıs como condiciones iniciales para $t=0$, $T=T_{0}$, puide ponerse como

$$
T=T_{a}+\left(T_{0}-T_{a}\right) e^{\cdot k t}
$$

es decir, una exponencial decreciente asintóticamente a la temperatura ambiente.

La forma logarítmica de esta ecuación sería

$$
\operatorname{Ln}\left(T-T_{a}\right)=k t+\operatorname{Ln}\left(T_{0}-T_{a}\right)
$$

\section{Calentamientos «naturales»}

De la misnla manera que hemos razonado para tratar un enfriamiento, un calentamiento «natural» (y esto no lo hemos visto tratado en ningún texto), es decir, el que sufre un cuerpo en un ambiente a mayor temperatura, debería obedecer también a una aley de calentamiento» simétrica a la del enfriamiento.

El desarollo matemático manteniendo las consideraciones sobre las constantes, sería ahora partir de Ia ecuación:

$$
\frac{d T}{\left(T_{n}-T\right)}=k d t
$$

y su solución sería:

$$
T=T_{a}-\left(T_{a}-T_{0}\right) e^{k t}
$$


que es una exponencial creciente asintóticamente a la temperatura ambiente.

La forma logarítmica de 7 es:

$$
\operatorname{Ln}\left(T_{a}-T\right)=k t+\operatorname{Ln}\left(T_{a}-T_{0}\right)
$$

Los resultados experimentales que presentamos a continuación confirman la validez de las ecuaciones 4 y 7 y nos atrevemos a decir que ponen en tela de juicio las afirmaciones acerca de la aproximación y validez de la ley de Newton ${ }^{1}$.

Al mismo tiempo, presentan lo que sería una «ley de calentamiento" que, así mismo, es experimentalmente confirmada.

\section{RESULTADOS EXPERIMENTALES}

Se utiliza como sistema de toma de datos el programa CASSY-E (Michael Hund, Leybold Didactic, 3.22, $1990 / 91$ ), suministrado por el MEC dentro del proyecto LAO, con los sensores de temperatura correspondientes. En todos los casos se utiliza la opción multímetro de dicho programa.

Este programa permite hacer la toma de datos de forma automática y la evaluación de los mismos de modo gráfico, permitiendo, si se desea, introducir una fórmula en su tratamiento. Nosotros lo utilizaremos para repre- sentarlos en forma logarítmica, pudiendo contrastar los resultados con la recta de ajuste a dicha gráfica. Permite, así mismo, hacer una representación múltiple (multigráfica) de datos de diversas experiencias, lo que hemos utilizado para ilustrar cualitativamente el comportamiento de los diferentes aislantes.

Las gráficas presentadas son las obtenidas por el programa.

\section{Estudio de los enfriamientos}

\section{Descripción}

En un vaso cilíndrico de acero $(9 \mathrm{~cm}$ de diámetro y $12 \mathrm{~cm}$ de altura) se colocan $500 \mathrm{~cm}^{3}$ de agua calientc y se introduce dentro de otro de plástico $(12 \mathrm{~cm}$ de diámetro y $13 \mathrm{~cm}$ de altura). El espacio entre ellos se rellena con diferentes tipos de aislantes. EI sistema va cerrado por una tapadera (que hemos aisiado en la experiencia 6) con un orificio para introducir el sensor. La toma de datos se realiza cada 60 segundos.

\section{Resultados obtenidos}

En la tabla 1 se especifican algunas de las experiencias realizadas, haciendo constar: el aislante utilizado, las temperaturas inicial y final del agua, el tiempo transcurrido en horas y la temperatura ambiente al principio y al final de la experiencia. En la columna de la derecha se especifica la temperatura ambiente media utilizada en la representación logarítmica de los datos obtenidos. (Todas las temperaturas vienen dadas en ${ }^{\circ} \mathrm{C}$ ).

Tabla I

\begin{tabular}{|c|c|c|c|c|c|c|c|c|}
\hline Aislante & Tipo de experiencia & $\begin{array}{l}\text { Núm. de } \\
\text { experiencia }\end{array}$ & $\mathrm{Ti}$ & Tf & $\mathrm{t}(\mathrm{h})$ & $\operatorname{Tam}_{i}$ & $\operatorname{Tam}_{\mathrm{f}}$ & $T_{a}$ \\
\hline Lana de vidrio & & 2 & 66,6 & 30 & 5 & 19,5 & 20,5 & 20 \\
\hline Termofix & 1 capa & 3 & 66,7 & 25,2 & 6,25 & 20,5 & 20,5 & 20,5 \\
\hline Termofix & 2 capas & 10 & 69,9 & 23 & 7,50 & 19,5 & 18,5 & 19 \\
\hline Aire & & 4 & 69,3 & 33,8 & 4,17 & 19,5 & 21 & 20,5 \\
\hline Burb. plast. & & 5 & 65,6 & 25,6 & 6,58 & 21 & 20 & 20,5 \\
\hline Lana de vidrio & $\begin{array}{l}\text { Tapa } \\
\text { aislada }\end{array}$ & 6 & 76,7 & 19,6 & 12 & 17 & 17 & 17 \\
\hline Ninguno & $\begin{array}{l}\text { Sin vaso } \\
\text { exterior }\end{array}$ & 7 & 69,6 & 28,2 & 3,75 & 18,5 & 19,5 & I9 \\
\hline Ninguno & Sin tapadera & 8 & 80,5 & 24,7 & 2,83 & 19 & 20 & 19,5 \\
\hline
\end{tabular}




\section{Evaluación gráfica}

A continuación se representan los datos obtenidos en algunas de las experiencias anteriores. A la izquierda se
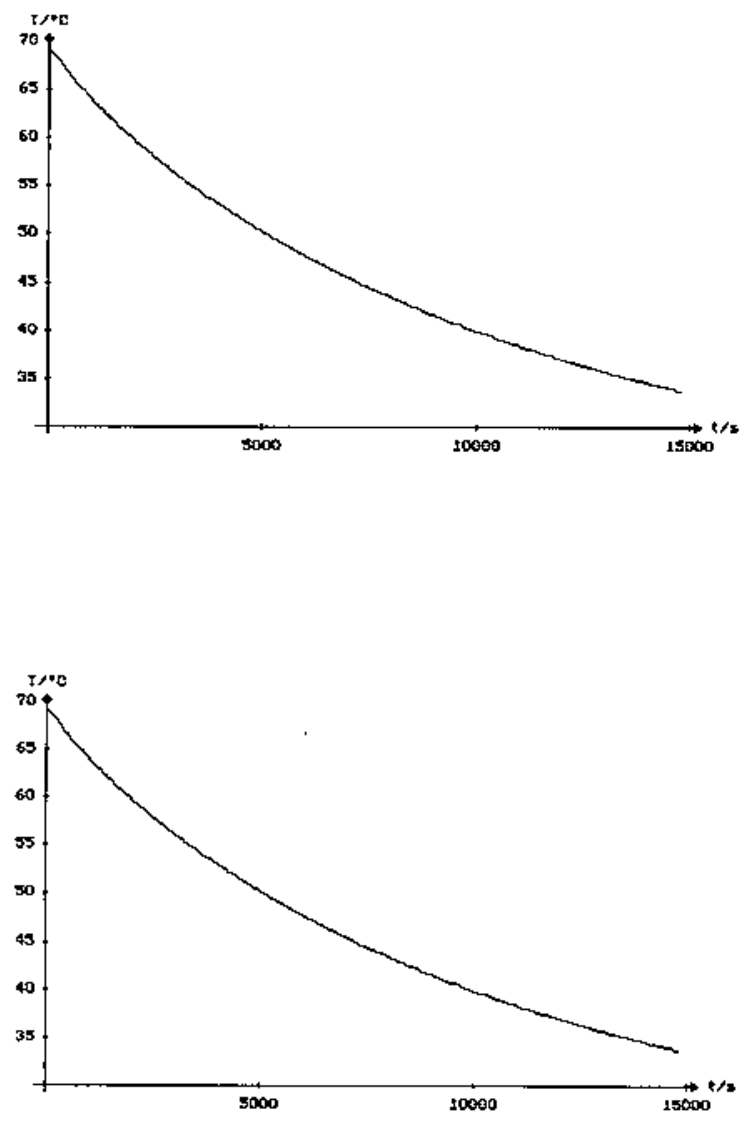

represent: a la temperatura (en ${ }^{\circ} \mathrm{C}$ ) frente al tiempo (en segundos). A la derecha el logaritmo neperiano de $T^{\prime}\left[\left(T-T_{a}\right)\right.$. Ver expresion (5)].
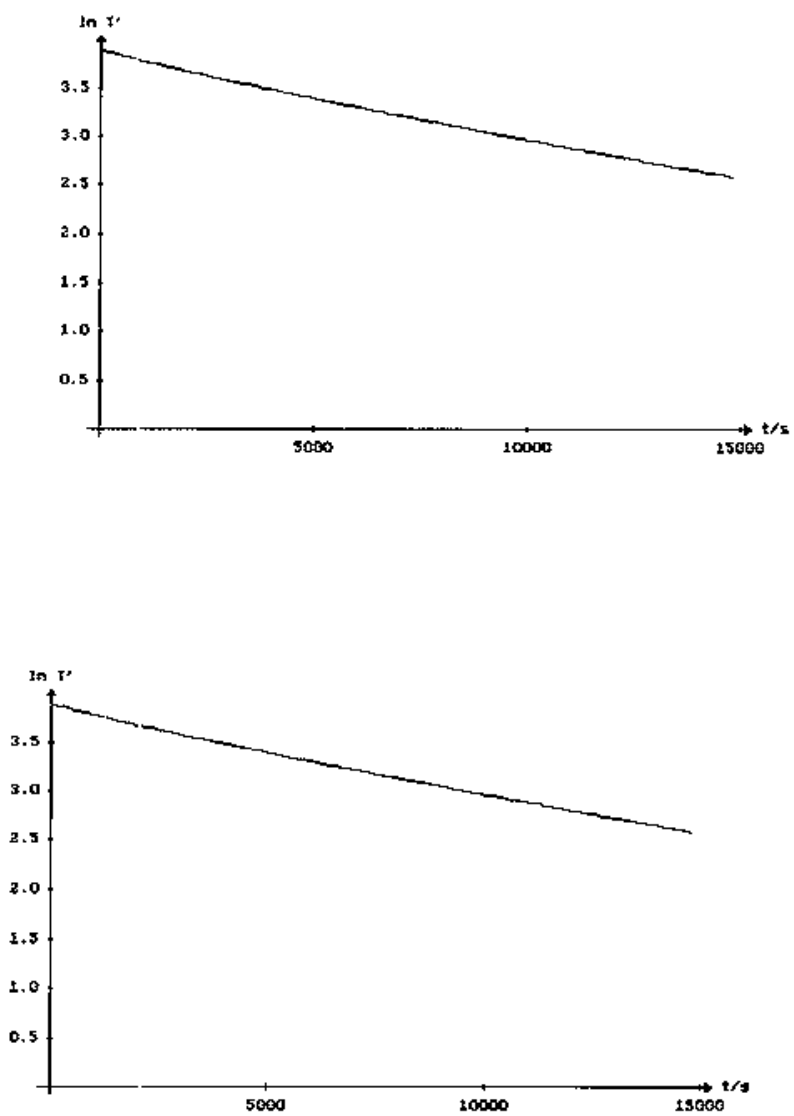

Experiencia 4
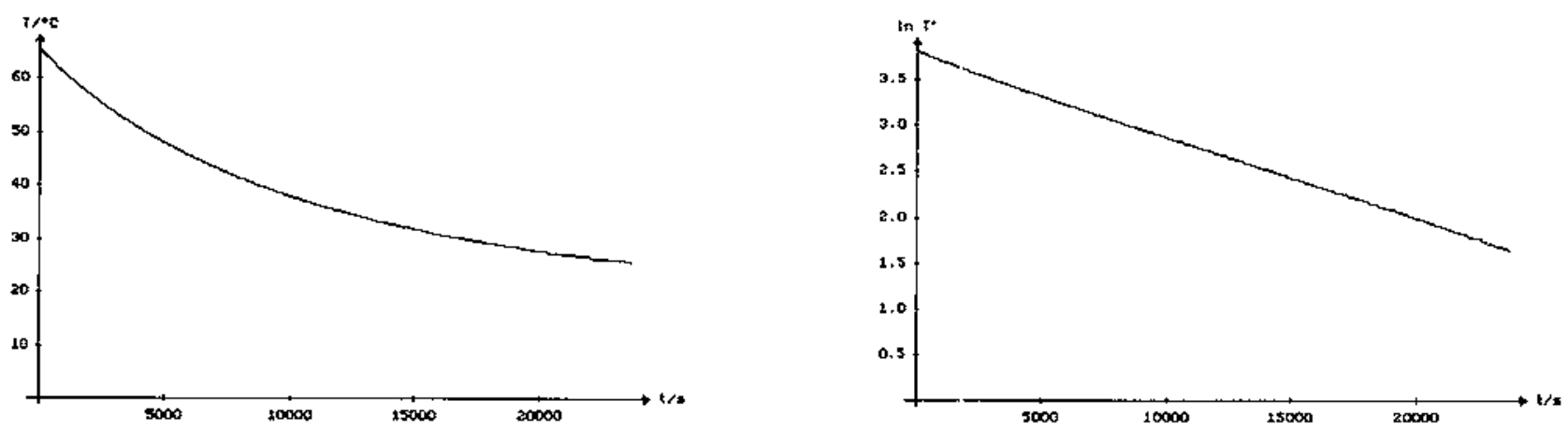

Experiencia 5 


\section{INVESTIGACIÓN DIDÁCTICA}
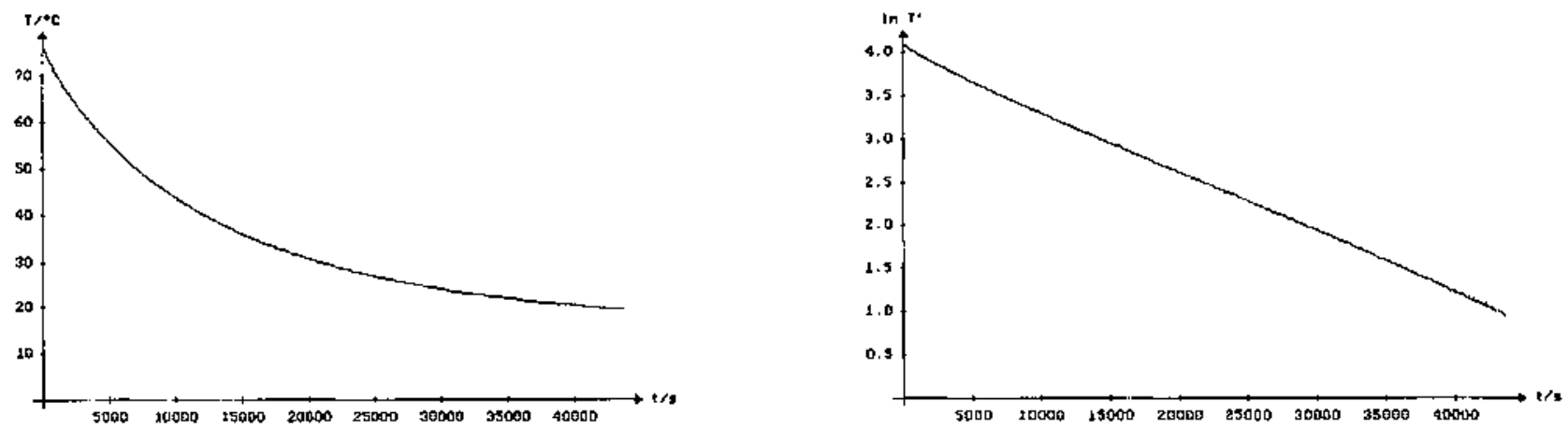

\section{Experiencia 6}

\section{Conclusiones}

De las gráficas anteriores se deduce que los enfriamientos siguen una ley que, sin duda, podemos considerar exponencial en todos los casos; tal como se predecía en Ia formula 4.

Las desviaciones al comportamiento exponencial son despreciables en casi todos los casos y pueden ser debidas a que, al haber sido la duración de los procesos de hasta $12 \mathrm{~h}$ (ver tabla 1), la temperatura ambiente variaba y Ia que hemos tomado para la expresión logarítrnica (formula 5) ha sido una media estimada. No creemos que la variación del calor específico en el rango de temperaturas manejado sea apreciable.

El caso en el que se aparta, apreciablemente, de dicho comportamiento es aquél en el que el enfriamiento se produce sin tapadera, con una temperatura inicial de $80,5^{\circ} \mathrm{C}$. Parece evidente pensar que ha sido debido a los efectos del cambio de estado en la evaporación, siendo, al principio, el descenso de temperatura mucho más pronunciado. En esta situacion, nos alejamos de las condiciones propuestas en el apartado anterior.

Para comprobarlo representaremos gráficamente el in ' $T$ ' de dicha experiencia (núm. 8) frente al tiempo, a la izquierda; $y$, a la derecha, la misma pero con recta de ajuste.

Por otro lado, y aunque no es éste el objetivo del trabajo, la influencia del aislante se puede ver en las dos gráficas siguientes, donde en multigráfica se representan en la de la izquierda los datos correspondientes a las experiencias 2 y 3 (la línea 1 corresponde a la experiencia núm. 2 y la 2 a la experiencia núm. 3). En la de la derecha, Ias experiencias 4,7 y 10 (las líneas 1,2 y 3 corresponden, respectivamente, a las experiencias 4,7 y 10 ).
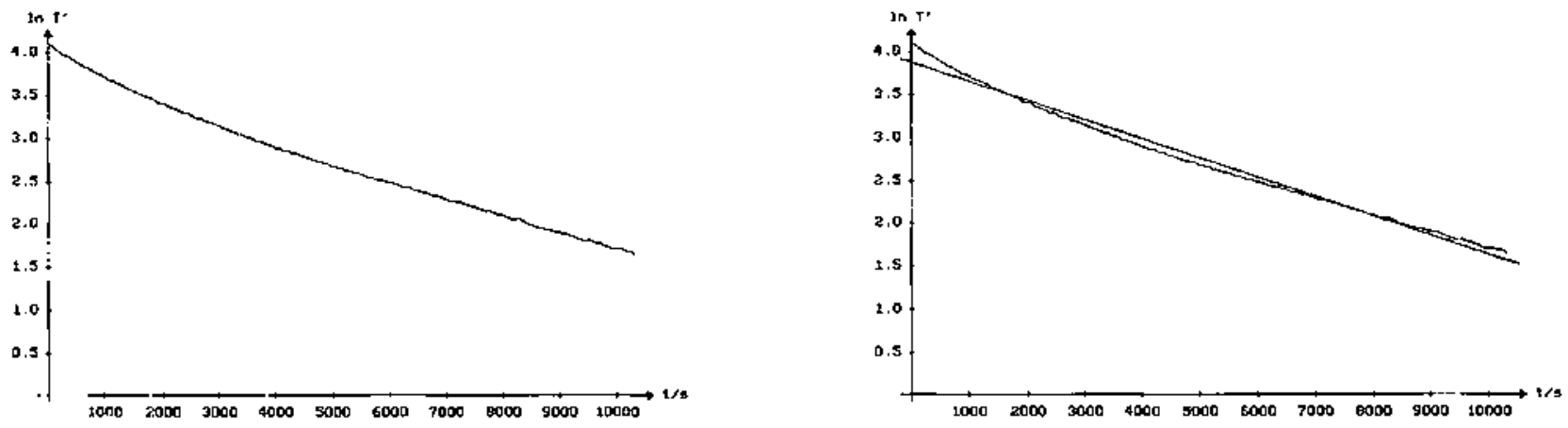


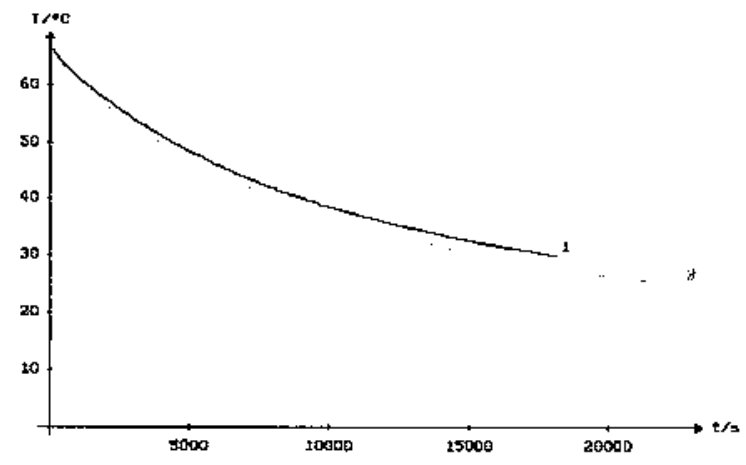

Experiencia 2 y 3

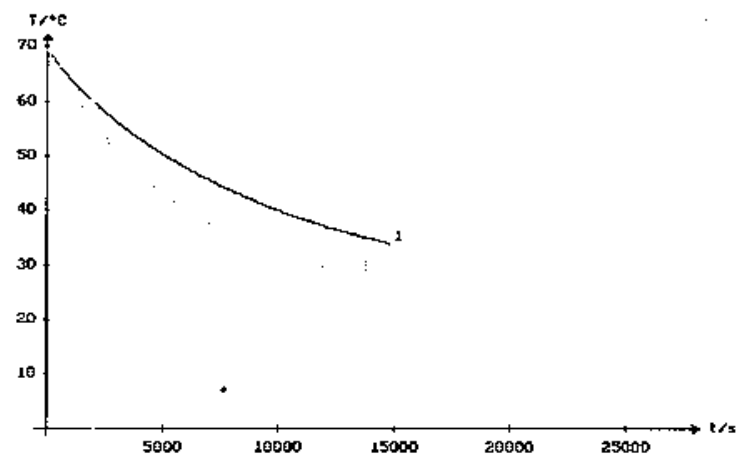

Experiencia 4,7 y 10

\section{Calentamientos por el medio ambiente}

Descripción

En el vaso metálico anteriormente utilizado, con tapadera, sin aisiar y «al aire», se colocan $250 \mathrm{~cm}^{3}$ de agua a la temperatura inicial indicada. En la experiencia 24 , la temperatura de $-6,1^{\circ} \mathrm{C}$ fue conseguida con una mezcla frigorífica de $\mathrm{NH}_{4} \mathrm{NO}_{3}$. La toma de datos se hace cada 60 seguncios.

\section{Resultados obtenidos}

\begin{tabular}{|l|c|c|c|c|c|c|}
\hline Núm. de experiencia & $\mathrm{Ti}$ & $\mathrm{Tf}$ & $\mathrm{t}(\mathrm{h})$ & $\operatorname{Tam}_{\mathrm{i}}$ & $\mathrm{Tam}_{\mathrm{f}}$ & $\mathrm{T}_{\mathrm{a}}$ \\
\hline Experiencia 22 & 8,8 & 19,4 & 8,58 & 20 & 20 & 20 \\
\hline Experiencia 23 & 9,7 & 19,8 & 6,83 & 20 & 20 & 20 \\
\hline Experiencia 24 & $-6,1$ & 19,9 & 5,33 & 21 & 20 & 20 \\
\hline
\end{tabular}

\section{Evaluación grafica}

Representación de los datos obtenidos en las experiencias anteriores. A la izquierda se representa la tempera- tura $\left(e^{\circ} \mathrm{C}\right.$ ) frente al tiempo (en segundos). A la derecha, el logaritno neperiano de $T$.
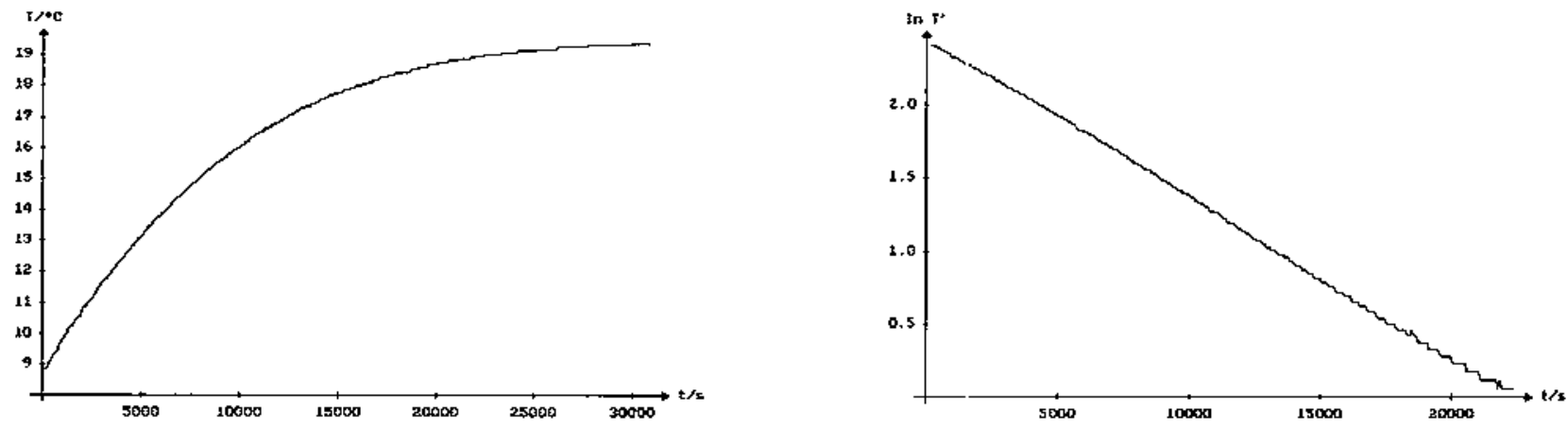

Experiencia 22 

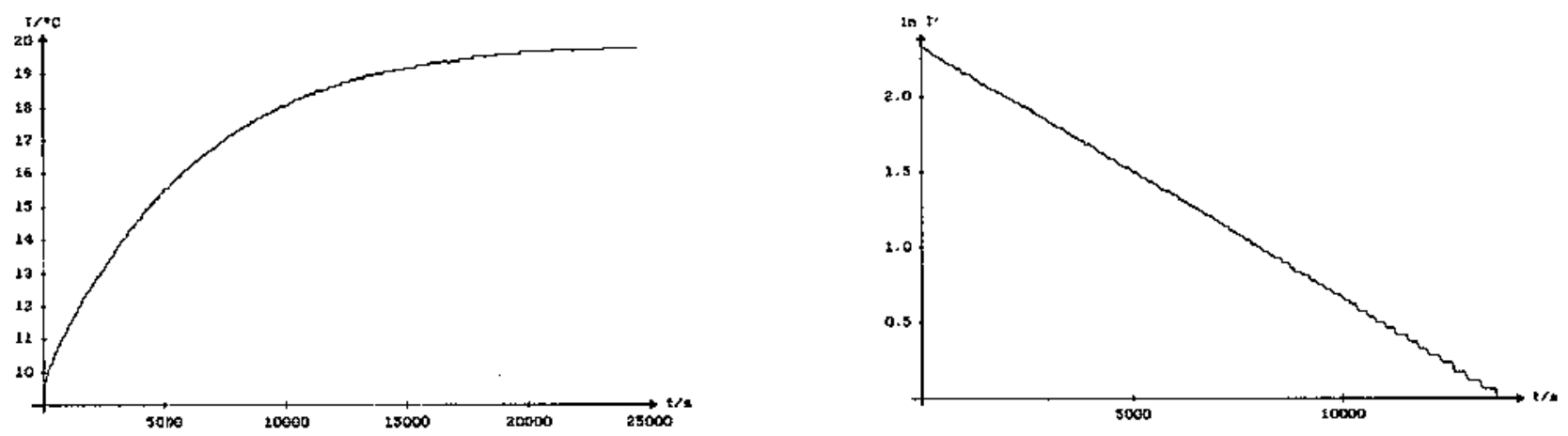

Experiencia 23
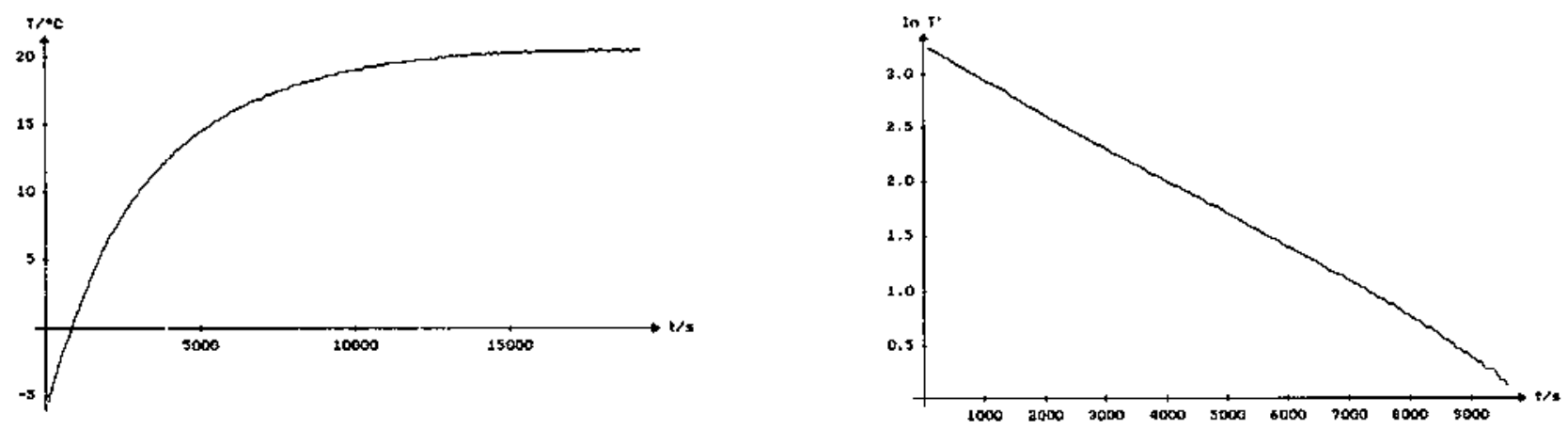

Experiencia 24

\section{Conclusiones}

Observando Ias gráficas, conchuimos que el calentamiento "naturai» (sin generador de energía calorífica) sigue una ley exponencial, tal como predice el análisis del apartado anterior y la expresión 7 .

\section{Calentamientos-enfriamientos}

\section{Descripción}

- El calentamiento por resistencia se realiza con $500 \mathrm{~cm}^{3}$ de agua colocados en el vaso metálico anterior introducido en el de plástico, aislados mediante lana de vidrio -incluida la tapadera-, mediante un calentador eléctrico de inmersión $(250 \mathrm{w} / 220 \mathrm{~V})$ conectado a 50 voltios para hacer más lento el proceso.

- El calentamiento en agua caliente se realiza con un vaso interior de aluminio (cilíndrico, de $7 \mathrm{~cm}$ diámetro y de $8 \mathrm{~cm}$ de altura) con tapadera y orificio conteniendo 250 $\mathrm{cm}^{3}$ de agua fría que ha sido introducido en un vaso exterior de plástico con $400 \mathrm{~cm}^{3}$ de agua caliente. (Uñ sensor solamente en el vaso interior del agua fría).
- Modos de enfriamiento utilizados:

- Conjunto: vaso interior introducido en el exterior y éste «aI aire».

- Exterior: vaso interior fuera del exterior y «al aire».

- En agua fría: vaso interior en un recipiente con 5 litros de agua fría a la temperatura de $12{ }^{\circ} \mathrm{C}$.

\section{Resultados obtenidos}

En la tabla siguiente se especifican algunas de las experiencias realizadas, haciendo constar: las temperaturas inicial, intermedia y final del agua; el tiempo transcurrido, en minutos, para el calentamiento y para el enfriamiento; y las temperaturas ambiente al principio y al final de la experiencia. Las dos primeras columnas corresponden al tipo de calentamiento (si es en agua caliente, se especifica la temperatura de esta) y enfriamiento.

(Todas las temperaturas vienen dadas en ${ }^{\circ} \mathrm{C}$ ).

En las experiencias 32 y 33 se utilizan $250 \mathrm{~cm}^{3}$ de agua fría en el vaso interior y $325 \mathrm{~cm}^{3}$ de agua caliente en el 
Tabla II

\begin{tabular}{|c|c|c|c|c|c|c|c|c|c|}
\hline Calentamiento & $\begin{array}{l}\text { Enfria- } \\
\text { miento }\end{array}$ & $\begin{array}{c}\text { Núm. de } \\
\text { experiencia }\end{array}$ & $\mathrm{Ti}$ & Tint & $\operatorname{tmin}(\mathrm{cal})$. & Tf & $\operatorname{tmin}(\mathrm{enf})$ & $\operatorname{Tam}_{\mathrm{i}}$ & $\operatorname{Tam}_{\mathrm{s}}$ \\
\hline Resistencia & Conjunto & 25 & 8,6 & 41,6 & 120 & 24,8 & 390 & 21 & 20,5 \\
\hline Resistencia & Conjunto & 26 & 19 & 45,1 & 107 & 20,2 & 713 & 19 & 19,5 \\
\hline Agua $90^{\circ} \mathrm{C}$ & Conjunto & 27 & 9,4 & 37,8 & 148 & 26,9 & 512 & 19 & 20 \\
\hline Agua $90^{\circ} \mathrm{C}$ & Exterior & 28 & 12,6 & 57 & 8,5 & 22,1 & 231 & $21^{\circ}$ & 21 \\
\hline Agua $60^{\circ} \mathrm{C}$ & Exterior & 29 & 22,3 & 43 & 12,5 & 30 & 73 & 21 & 21 \\
\hline Agua $60^{\circ} \mathrm{C}$ & Exterior & 30 & 22 & 41,9 & 13,8 & 23,8 & 120 & 19,5 & 19,5 \\
\hline Agua $65^{\circ} \mathrm{C}$ & Exterior & 32 & 10,8 & 40 & 12,8 & 22,3 & 180 & 20 & 20 \\
\hline Agua $67^{\circ} \mathrm{C}$ & Agua a $12^{\circ} \mathrm{C}$ & 33 & 9,2 & 49,1 & 14 & 16,5 & 31 & 20,5 & 20,5 \\
\hline
\end{tabular}

exterior. En las experiencias 25,26 y 27 , la toma de datos se hace cada 60 segundos y, en las restantes, cada 15 segundos, en la opción multímetro.

Como se puede observar, el tiempo que tarda en calentarse el agua mediante un foco calorífico es mucho menor que el enfriamiento. De ahí que optásemos por disminuir la potencia calorífica reduciendo el voltaje de la corriente y, posteriormente, por enfriar sin aislante y en agua fría, para que los tiempos se acercasen lo más posible.

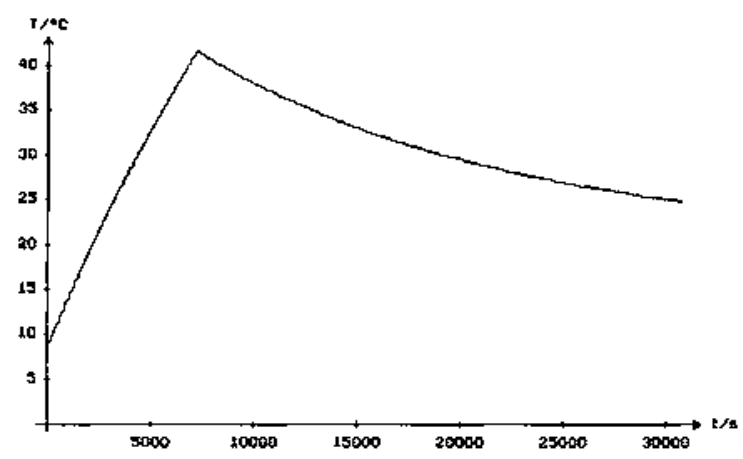

Experiencia 25
En la experiencia 27, el vaso interior estaba aislado con dos capas de aislante termofix, de ahí que el tiempo que tarda en calentarse, 148 minutos, sea mucho mayor que en la experiencia 28 , en la cual está sin aislar y tarda 8,5 minutos.

\section{Evaluación gráfica}

A contintación se representan los datos obtenidos en alguna di las experiencias anteriores: temperatura (en ${ }^{\circ} \mathrm{C}$ ) frente al tiempo (en segundos).

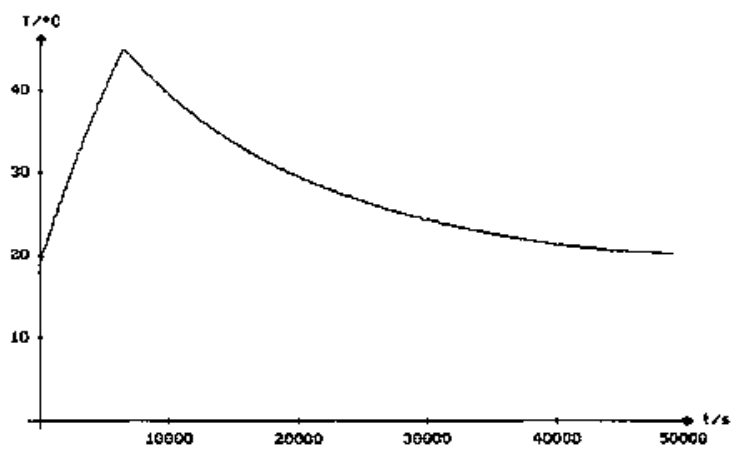


ANEXO

1. Los procesos en los que un cuerpo varía su temperatura se denominan de «calentamiento» o de «enfriamiento».

¿Podrias decir cuáles son las variables de qué depende la variación de temperatura experimentada por un cuerpo en tun proceso de enfriamiento o de calentamiento?

¿Recuerdas cuál es la relación matemática existente entre el calor $Q$, y la variación de temperatura $\Delta \mathrm{T}$ ?

2. La gráfica que te presentamos representa el calentamiento en el laboratorio, mediante un mechero de butano, de una determinada cantidad de agua.

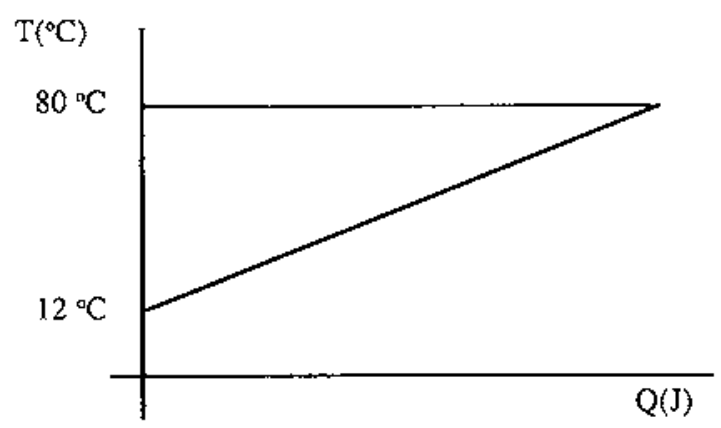

Una vez alcanzada la temperatura de $80^{\circ} \mathrm{C}$, se apaga el calentador de butano y se deja que el agua calentada se enfríe. Se toman medidas de la temperatura cada cierto intervalo de tiempo mientras dura el enfriamiento. A continuación se representa gráficamente ta temperatura frente al tiempo.

¿Cómo sería la gráfica que correspondería a este proceso de enfriamiento?

3. El agua que sale del grifo, en invierno, está a una temperatura de unos $10^{\circ} \mathrm{C}$. Ponemos $250 \mathrm{~cm}^{3}$ de dicha agua en un vaso y la dejamos en el laboratorio, cuya temperatura ambiente es de unos $18^{\circ} \mathrm{C}$, prácticamente constante.

Metemos un termómetro en el vaso y anotamos la temperatura cada cierto tiempo.

¿Cual será la temperatura final del agua en el vaso?

Si representamos con una grática la evolución de esta temperatura del agua frente al tiempo, ¿cómo sería la gráfica que se obtendria?

4. Para determinar el cator específico $(C e)$ del aluminio por el procedimiento de las mezclas, se calienta una pieza de aluminio al baño maría hasta una temperatura de $75^{\circ} \mathrm{C}$ y se introduce a continuación en un vaso calorimétrico que contiene agua a $15^{\circ} \mathrm{C}$. Al cabo de 15 minutos el sistema alcanza la temperatura de equilibrio, que resulta ser de $22^{\circ} \mathrm{C}$.

Representa gráficamente, en una única gráfica, la variación de temperatura experimentada por el aluminio y por el agua con el tiempo.

5. Los mismcs $250 \mathrm{~cm}^{3}$ de agua del grifo $\left(10^{\circ} \mathrm{C}\right)$ de la cuestión tercera, los calentamos ahora con un hornillo hasta que alcancen la temperatura ambiente de 18 "C.

¿Qué diferencia encontraríamos en relación a lo que dijiste que pasaba entonces?

Representa en una gráfica única los dos calentamientos en función del tiempo. 